\title{
A Novel 2D Feature Extraction Method for Fingerprints Using Minutiae Points and Their Intersections
}

\author{
Nibras Ar Rakib, SM Zamshed Farhan, Md Mashrur Bari Sobhan, Jia Uddin, Arafat Habib \\ Department of Computer Science and Engineering, BRAC University, Dhaka, Bangladesh
}

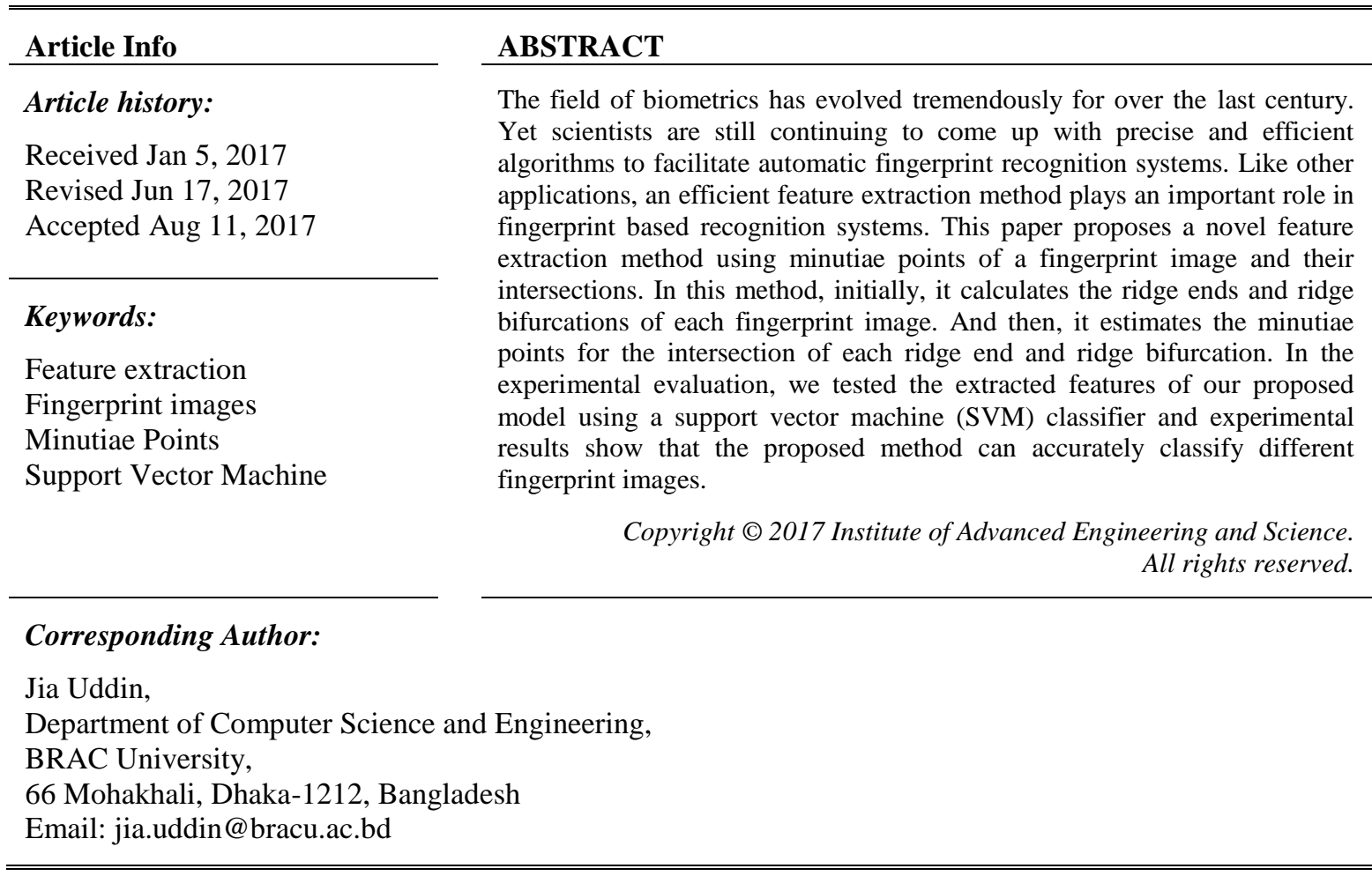

\section{INTRODUCTION}

The potential of the use of fingerprints has long been recognized as a fundamental one. One of the earliest instances of this can be found in the contributions of John Edgar Hoover, the first Director of the Federal Bureau of Investigation, when he created one of largest databases of fingerprints to date. As such, fingerprint has become a common reference point for identifying a human being, since it happens to be unique for a person.

Fingerprints, when analyzed, reveal uneven flow of their unique patterns called ridges. A ridge is defined as a single curved segment, and a valley is the region between two adjacent ridges [1]. The pattern of ridges also consists of uneven discontinuities at several points, known as minutiae points. The potential of this phenomenon was realized when Chi-Jim [2] showed a smooth classification process, a thorough analysis of fingerprints, its indexing procedures and how these can be used later to identify a human being [3-5].

The minutiae features are considered to be a major feature of biometrics and include several partsridge endings, ridge bifurcations, island, core, delta, bridge or crossover, ridge enclosure, short ridge, spur, etc. But, for the purpose of identification using fingerprints mostly only two of these features are significant: ridge endings and ridge bifurcations, as shown in Figure 1. Ridge bifurcations are the points where a ridge gets divided into two and ridge endings are those points where the ridges get terminated. These features are unique for a human being and thus help in the identification and verification processes. 


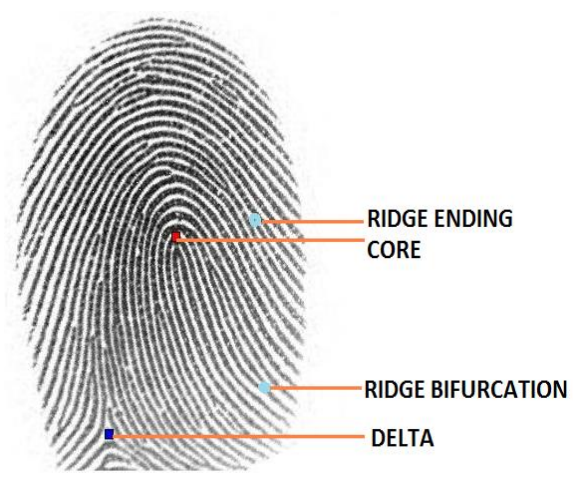

Figure 1. Fingerprint image showing ridges, ridge endings and ridge bifurcations.

There are two steps involved with matching: registration and verification. In the registration step, all fingerprints of a set of person are captured, processed and stored as a template which is much smaller than the original image, in a database for later use [6-7]. In the verification step, a person gives his fingerprint to verify the identity- the fingerprint is compared with the ones stored in the database [3].

In this paper, a set of fingerprints of different personnel is chosen at random and the ridge ending points along with the bifurcation points are found out from the fingerprints. These are then processed further using filtering technique, namely single-level 2-D Discrete Wavelet Transform (DWT), to find an increased number of points and thus optimizing the whole procedure. They are then plotted and joined to make separate best fit curves. The points where the curves intersect are found. This paper proposes that these intersection points are unique for different human beings and the assumption is proven further through the use of clustering. Finally, the machine learning technique, Support Vector Machine (SVM) is used to classify the fingerprints efficiently. However, the recent performance comparison in the area of finger print depends on how far the accyracy, efficacy and scalability performance can be increased [8-13].

Fingerprint image segmentation is an important pre-processing step in automatic fingerprint recognition system and a well-designed fingerprint segmentation technique can improve the accuracy in collecting clear fingerprint area and mark noise areas [14]. To overcome the limitation of the works mentioned above, A Novel 2D Feature Extraction Method for Fingerprints Using Minutiae Points and Their Intersections has been prposed in this article. The rest of the paper is organized as follows - Section II provides a detailed overview of the steps of the proposed feature extraction method, Section III includes the results of the experiments carried out and their analysis and Section IV concludes the paper.

\section{PROPOSED FEATURE EXTRACTION METHOD}

To carry out the extraction of features and the classifications a set of steps has been carefully designed. A block diagram of proposed feature extraction method is presented in Figure 2.

To validate the proposed model, a set of fingerprint images is taken from the internet and then converted into gray level images witha size $300 \times 300$. The image is then processed using an image processing technique, which is clearly describe in the following sub-section A. This is done to make sure that the minutiae features of the fingerprint are very much visible, as it can then be fed to other processes as per requirements. In this paper, seven fingerprint images were used to test our proposed model.

\subsection{Image Processing}

For a fingerprint image, a portion of the fingerprint is first selected and cropped, as doing otherwise would produce too much information. On that particular selection, image processing technique is applied, which would ultimately convert the image into its binary format, where each pixel is represented by 1-bit. The binary image of the fingerprint is then further thinned to reveal the minutiae points. The ridge ends and the ridge bifurcations are pointed out, as depicted in Figure 3, and in the next section these are filtered to optimize results.

\subsection{Applying 2-D Wavelet Transform}

As mentioned earlier, to optimize the process of finding out the minutiae features of the fingerprint images they are filtered using some filtering techniques. At first the single-level 2-D DWT was applied on the image. The 2-D wavelets are used in a number of areas, most notably in image manipulation and helps 
with de-noising [10]. 2-D wavelet works with approximation coefficients matrix- $c A$ and details coefficients matrices- $c H, c V$, and $c D$ (horizontal, vertical, and diagonal), but we have only used the vertical one, $c V$ [6]. This is because, while high-frequency components can capture discontinuities, ruptures and singularities in the original data, low-frequency components characterize the coarse structure of the data, to identify the long-term trends in the original data [9].

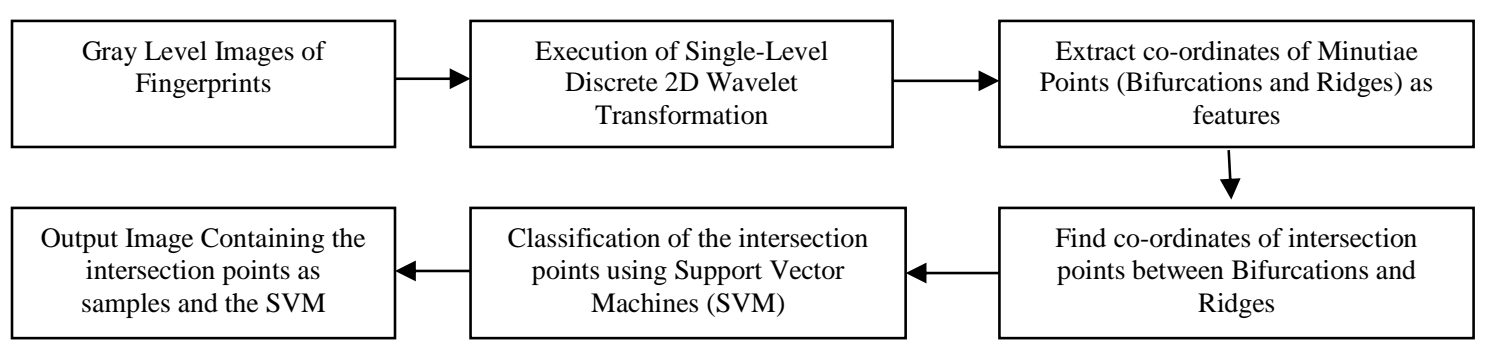

Figure 2. Block diagram consisting of the steps followed for the feature extraction and verification.

\subsection{Plots and Intersecting Points}

In the next step the produced ridge ends and the bifurcations are plotted separately. The points on each of the bifurcation and ridge end plots are then joined together to produce a best fit line for each of them. While we are finding bifurcation point from image, we represent them as row and column. From image, bifurcation points returned as the row and column subscript of nonzero element. Same procedure follows to plot ridge. These two plots are then mapped onto each other, as shown below, Figure 5, and merged to find out the intersecting points between them.

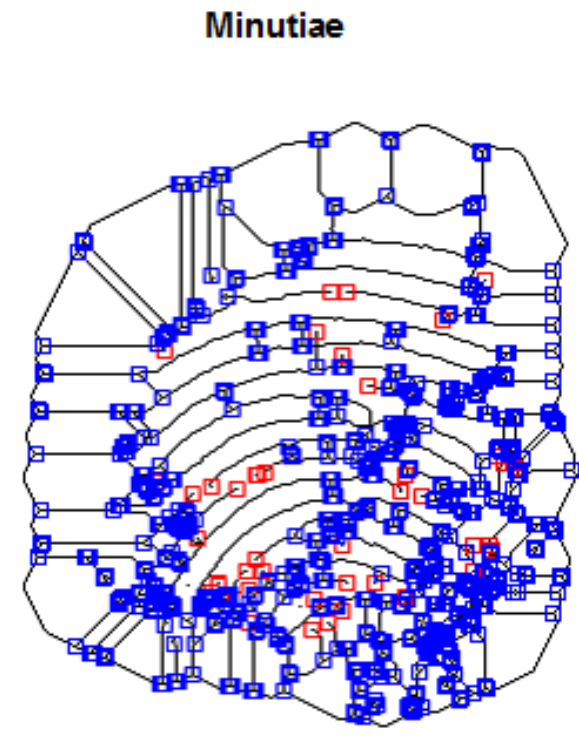

Figure 3. Fingerprint with ridge ends (red), and ridge bifurcations (blue).

The row and column number is represent for each bifurcation and ridge andit is representedin $\mathrm{X}$ and $\mathrm{Y}$ axis respectively.During the finding of intersection point between bifurcation and ridge, Bifurcation-X and Ridge- $\mathrm{X}$ represent $\mathrm{X}$ axis and Bifurcation- $\mathrm{Y}$ and Ridge- $\mathrm{Y}$ represent $\mathrm{Y}$ axis. The purpose of it to find out the intersecting points between the lines produced from the ridge ends and ridge bifurcations.It is noticeable that the intersecting points ofdifferent fingerprints are unique.

\subsection{Clustering and Verification}

Using the points of intersections found in the earlier section, a plot is made where both sets of points reside. Figure 6 illustrates an overview to examine whether the points reside within distinct clusters, contains 
data-points from separate fingerprints accumulated together. As depicted in Figure 6, the plot does not produce a clear clustering image. So, the same cluster is reproduced for a particular range, 80-150(horizontal) and 70-150 (vertical), which gives a clearer clustering image, as shown in Figure 7.From the plot, it is very much evident that, the intersecting points from different fingerprints make separate clusters distinctively, meaning that the intersecting points from the ridge ends and ridge bifurcations are unique and therefore it is expected that it can used as a good feature of a fingerprint image. It is important to classify different personnel using these particular features through the use of some method, a machine learning technique in our case, which the following section will describe thoroughly.



Figure 4. After Single-level discrete 2-D Wavelet transform, fingerprint with ridge ends (red), and ridge bifurcations (blue)

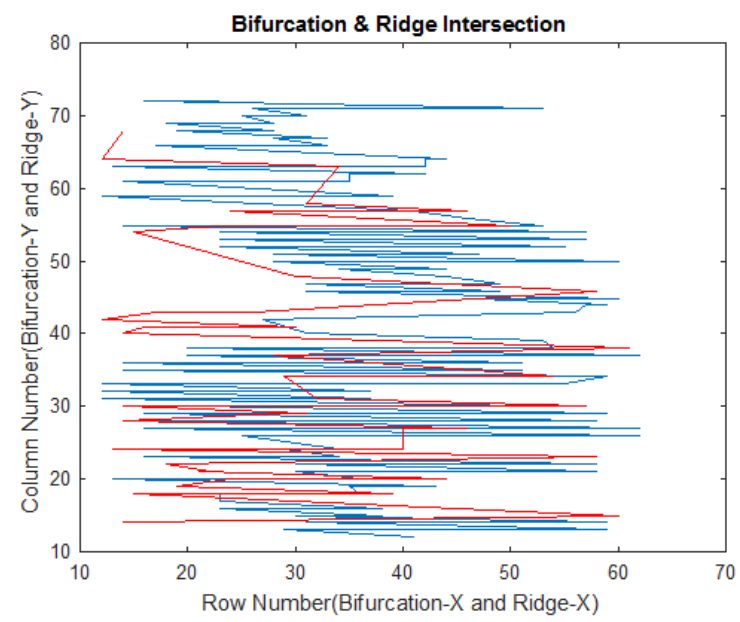

Figure 5. Lines joining the bifurcations and the ridge endings are drawn onto the same plot to find out the intersecting point. 


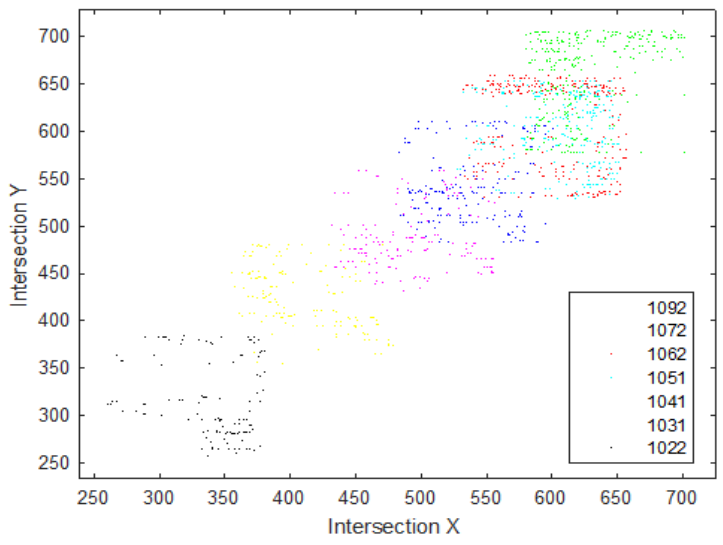

Figure 6. Different coloured points represent data-points from different fingerprint images.

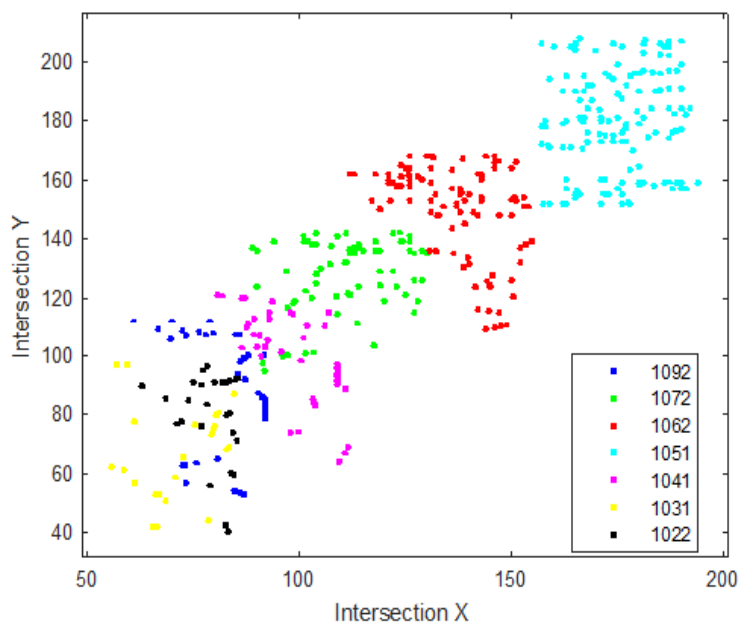

Figure 7. Previous clustering picture for a particular range, 80-150 (horizontal) and 70-150 (vertical), to get a clearer clustering image.

\subsection{Classification of Biometrics}

To classify different sets of fingerprint features it is important to use a machine learning technique. In this paper, the Support Vector Machine (SVM) was utilized [11,12]. The SVM is a learning machine for two-group classification problems [4]. Generally, an SVM creates a hyper plane or a set of hyper planes that can be used for classification and regression. The hyper plane takes a form of the equation:

$$
f(x)=w \cdot x+b
$$

where, $w$ is the weight vector, $x$ is the data and $b$ is called the bias [8].

To classify a set of points, they are put as $x$ into a decision function:

$$
\mathrm{D}(\mathrm{x})=\operatorname{sign}(\mathrm{w} \cdot \mathrm{x}+\mathrm{b})
$$

\section{EXPERIMENTAL RESULTS AND ANALYSIS}

It is important to note that, the significant results for this whole experiment is provided by the SVM classification. For starter, the SVM was trained using two groups, each containing the features obtained from a person's fingerprint. The produced results for the classification through SVM are presented in Figure 8. The same method was then used to let the SVM classify two different fingerprint features by itself, and from the results produced. In Figure 9, it can be seen that, these two produced plots do not differ much. 
These previous steps are then repeated using one fingerprint features on one group and seven fingerprint features together on the other to train the SVM, as shown in Figure 10. The same method is applied to let the SVM classify a different, but same number on each group, set of fingerprint features, and again the results produced as given in Figure 11 do not differ much. The produced results show promising classifications of separate fingerprints using the intersection of the bifurcation and ridge end features of those fingerprints.

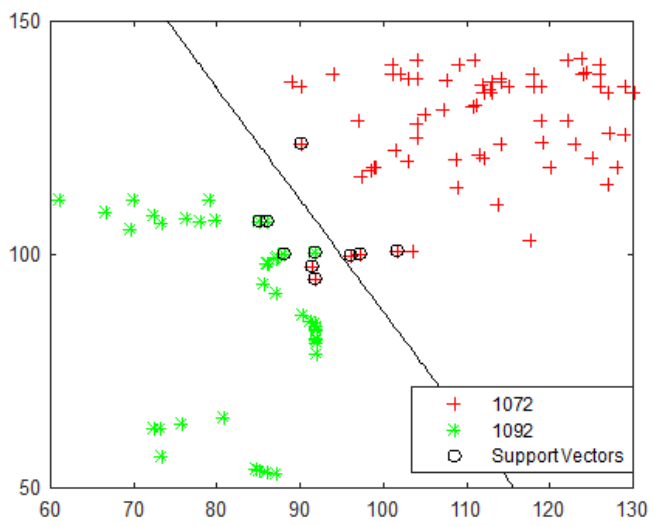

Figure 8. SVM training using two fingerprint features.

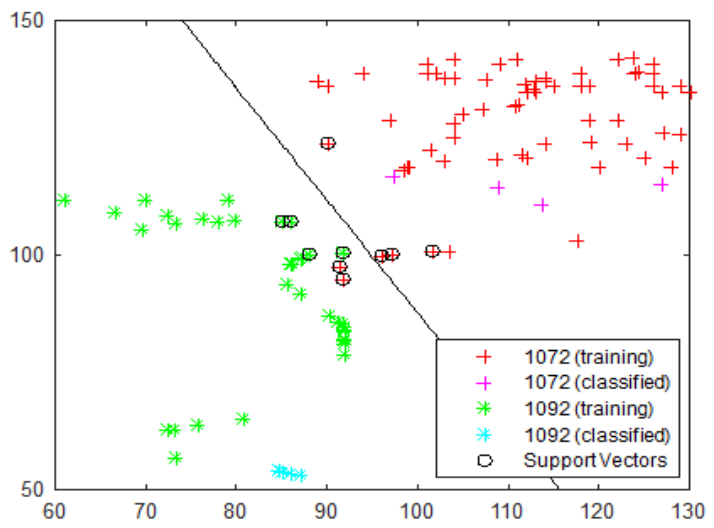

Figure 9. SVM classification Plot using two separate fingerprint features.

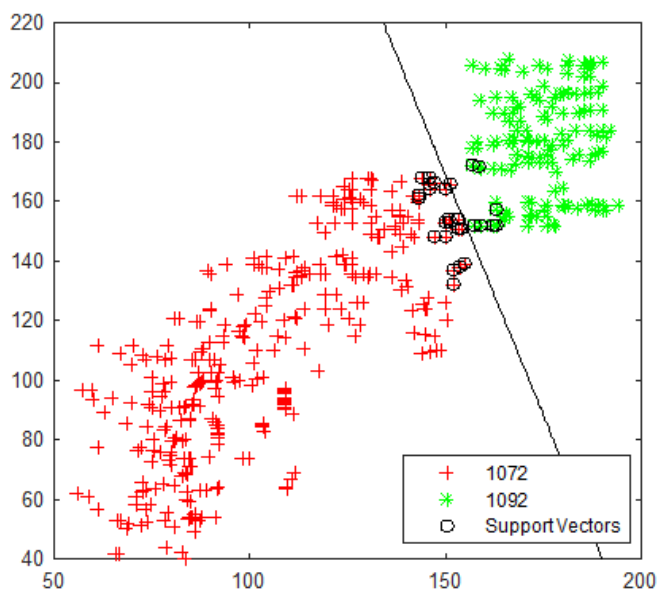

Figure 10. SVM training Plot using one fingerprint features on one group and seven fingerprint features on the other. 


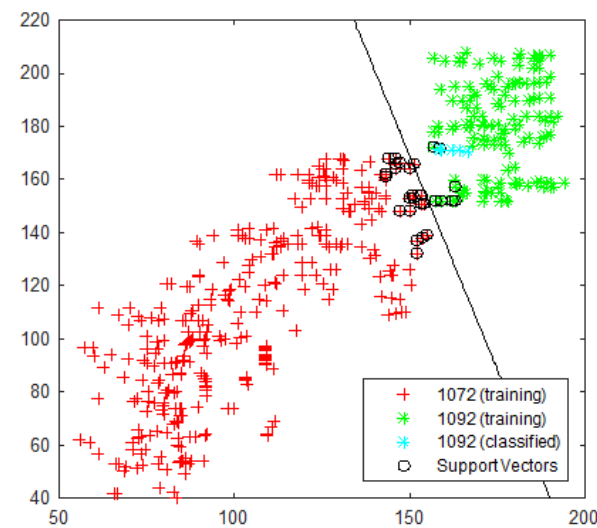

Figure 11. SVM training Plot using one fingerprint features on one group and seven fingerprint features on the other.

\section{CONCLUSION}

This paper proposed a new 2D feature extraction method for fingerprint images. In this method, they extracted unique feature of each fingerprint image using the intersection of its ridge ends and ridge bifurcations. Finally, the classification accuracy of proposed method was tested for a dataset using a support vector machine classifier. According to SVM results, the capability of the minutiae extracting and matching has been proved in experimental results. In spite of a greater conceptual complexity, it has been shown that this technique has less computational complexity at the classification part as fewer points are needed to classify within the SVM.

\section{REFERENCES}

[1] R. Thai, "Fingerprint Image Enhancement and Minutiae Extraction," Report of HonoursProgramme of the School of Computer Science and Software Engineering, The University of Western Australia, 2003.

[2] C. Chi-Jim, P. Tun-Wen, and C. Mox, "A Support Vector Machine Approach for Truncated Fingerprint Image Detection from Sweeping Fingerprint Sensors,” International Journal of Sensors, 2015, vol. 15, pp. 7807-7822.

[3] S.M. Mohsen, S.M.Z. Farhan, and M.M.A. Hashem, "Automated Fingerprint Recognition: Using Minutiae Matching Technique for The Large Fingerprint Database," in Proc. of 3rd International Conference on Electrical \& Computer Engineering, ICECE 2004, 28-30 December 2004, Dhaka, Bangladesh.

[4] C. Cortes, and V. Vapnik, "Support-vector networks," Machine Learning, 1995, vol. 20, no. 3, pp. 273.

[5] A. Jain, A. Ross, and S. Prabhakar, "Fingerprint Matching Using Minutiae And Texture Features," in Proc. of International Conference on Image Processing, ICIP 2001, pp. 282-285, Oct 7 - 10, 2001, Thessaloniki, Greece.

[6] Single-level discrete 2-D wavelet transforms. [Online]. [Accessed 31 November 2015]. Online. http://www.mathworks.com/help/wavelet/ref/dwt2.html

[7] P.D. Sirivella, and D.R Vamsi, "Fingerprint Validation and Outlier Detection Using Minutiae Approach in Network Security," International Journal of Computer and Organization Trends, 2012, vol. 2, no. 5, pp. 123-127.

[8] A Yuniarti, "Classification and numbering of dental radiographs for an automated human identification system," TELKOMNIKA Telecommunication, Computing, Electronics and Control., vol. 10, no. 1, pp. 137-146, 2012.

[9] S. Lahmiri, "Wavelet low- and high-frequency components as features for predicting stock prices with backpropagation neural networks," Journal of King Saud University - Computer and Information Sciences archive, July 2014, vol. 26, no. 2, pp. 218-227.

[10] F. Xiao, and Y. Zhang, "A comparative study on thresholding methods in wavelet-based image denoising," Procedia Engineering, 2011, vol. 15, no. 0, pp. 3998-4003.

[11] J. Uddin, D. Nguyen and J. Kim, "A Reliable Fault Detection and Classification Model of Induction Motors using Texture Features and Multi-class Support Vector Machines," Journal of Mathematical Problems in Engineering, Hindawi, United States, vol. 2014 (2014), article ID 814593, pp. 1-9.

[12] J. Uddin, R. Islam and J. Kim, "Texture Feature Extraction Techniques for Fault Diagnosis of Induction Motors," Journal of Convergence, FTRA, South Korea, vol. 5, no. 2, June 2014, pp. 15-20.

[13] G. Indrawan, S. Akbar and B. Sitohang, "Fingerprint Direct-Access Strategy Using Local-Star-Structure based Discriminator Features: A Comparison Study," International Journal of Electrical and Computer Engineering vol. 4, no.5, October 2014, pp. 817-830.

[14] S. Saparudin, S. Akbar and G. Sulong, "Segmentation of Fingerprint Image Based on Gradient Magnitude and Coherence," International Journal of Electrical and Computer Engineering vol.5, no.5, October 201, pp. 834-849. 


\section{BIOGRAPHIES OF AUTHORS}
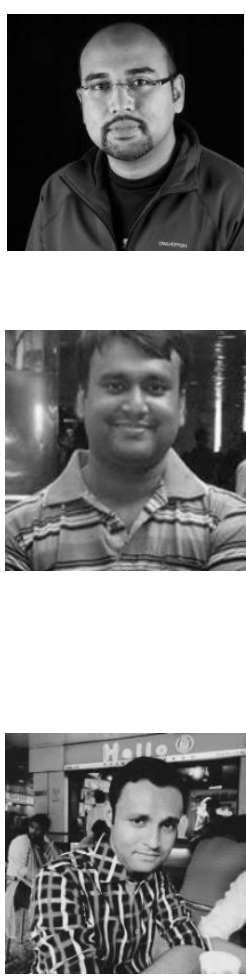

Md. Mashrur Bari Sobhan, is a Graduate Student at Department of Computer Science and Engineering in BRAC University, Dhaka, Bangladesh. He received Bachelor of Engineering (BEng), Electrical and Electronics Engineering, Queen Mary, U. of London, UK. Mr. Mashrurworked on the development of a web application for the Local Government Engineering Department (LGED) of Bangladesh to record survey and roads' information for further analysis. Responsibilities included designing the database systems along with deciding on the independent functionalities of the web application, implementation of ASP. NET MVC framework with the support of SQL Server Management Studio for the purpose of web developments and maintaining a liaison with foreign consultants and LGED to facilitate the developments at every step. His research areas are database, image processing and machine learning.

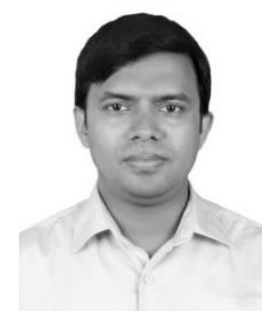

Dr. Jia Uddin, is an Assistant Professor of Computer Science and Engineering Department of BRAC University. He was an Assistant Professor in Department of Computer and Communication Engineering Department in International Islamic University Chittagong, Bangladesh. He received Ph.D. degree (Computer Engineering) from University of Ulsan, South Korea in January 2015. During his Ph.D. duration (2011-2014), he was involved with a research laboratory "Embedded Ubiquitous Computing System Lab" and has a number of peer reviewed journals. He attended several international conferences and symposiums at home and abroad. Prior to his Ph.D., he obtained M.Sc. Engg. (Telecommunications) degree from Blekinge Institute of Technology, Sweden at 2010. He received B.Sc. Engg. in Computer and Communication Engineering from International Islamic University Chittagong, Bangladesh at 2005. His research interests include Parallel Computing, Fault Diagnosis and Ad-Hoc Networks.

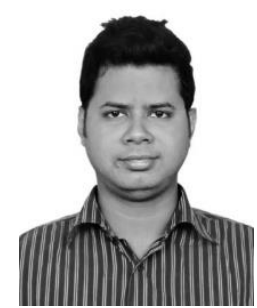

Arafat Habib has completed B.Sc in Computer Science from BRAC University very recently. His research interest lies in Cloud Computing, Reinforcement Learning, Robotics and Supply Chain Management. Previously, he worked as a Database Administrator and Java developer in his student life. 